\title{
Rotational spectroscopy and astronomical search for glutaronitrile ${ }^{\star}$
}

\author{
C. Cabezas ${ }^{1}$, C. Bermúdez ${ }^{1}$, Y. Endo ${ }^{2}$, B. Tercero ${ }^{3,4}$, and J. Cernicharo ${ }^{1}$ \\ ${ }^{1}$ Instituto de Física Fundamental (IFF-CSIC). Group of Molecular Astrophysics, C/ Serrano 123, 28006 Madrid, Spain \\ e-mail: carlos.cabezas@csic.es \\ 2 Department of Applied Chemistry, Science Building II, National Chiao Tung University, 1001 Ta-Hsueh Rd., Hsinchu 30010, \\ Taiwan \\ 3 Centro de Desarrollos Tecnológicos, Observatorio de Yebes (IGN), 19141 Yebes, Guadalajara, Spain \\ ${ }^{4}$ Observatorio Astronómico Nacional (OAN-IGN), C/ Alfonso XII 3, 28014 Madrid, Spain
}

Received 19 February 2020 / Accepted 3 March 2020

\begin{abstract}
Context. Nitriles constitute almost $15 \%$ of the molecules observed in the interstellar medium (ISM), surprisingly only two dinitriles have been detected in the ISM so far. The lack of astronomical detections for dinitriles may be partly explained by the absence of laboratory rotational spectroscopic data.

Aims. Our goal is to investigate the rotational spectrum of glutaronitrile, $\mathrm{N} \equiv \mathrm{C}-\mathrm{CH}_{2}-\mathrm{CH}_{2}-\mathrm{CH}_{2}-\mathrm{C} \equiv \mathrm{N}$, in order to allow its possible detection in the ISM.

Methods. The rotational spectrum of glutaronitrile was measured using two different experimental setups. A Fourier transform microwave spectrometer was employed to observe the supersonic jet rotational spectrum of glutaronitrile between 6 and $20 \mathrm{GHz}$. In addition, the $\mathrm{mmW}$ spectrum was observed in the frequency range $72-116.5 \mathrm{GHz}$ using a broadband millimetre-wave spectrometer based on radio astronomy receivers with fast Fourier transform backends. The spectral searches were supported by high-level ab initio calculations.

Results. A total of 111 rotational transitions with maximum values of $J$ and $K_{a}$ quantum numbers 54 and 18 , respectively, were measured for the $g g$ conformer of glutaronitrile. The analysis allowed us to accurately determine the rotational, nuclear quadrupole coupling, quartic and sextic centrifugal distortion constants. These rotational parameters were employed to search for glutaronitrile in the cold and warm molecular clouds Orion KL, Sgr B2(N), B1-b and TMC-1, using the spectral surveys captured by IRAM $30 \mathrm{~m}$ at $3 \mathrm{~mm}$. Glutaronitrile was not detected, and the upper limits' column densities were derived. Those are a factor of 1.5 and 5 lower than those obtained for the total column densities of the analogous succinonitrile in Orion KL and Sgr B2, respectively.
\end{abstract}

Key words. ISM: molecules - methods: laboratory: molecular - molecular data - line: identification

\section{Introduction}

The chemical complexity of the interstellar medium (ISM) has been demonstrated by detecting more than 200 different chemical species ${ }^{1}$. The list of observed molecules includes very simple species like methylidyne $(\mathrm{CH})$, the first molecule detected in the ISM (Swings \& Rosenfeld 1937; Dunham 1937; McKellar 1940, or more complex structures like $c-\mathrm{C}_{6} \mathrm{H}_{5} \mathrm{CN}$ (McGuire et al. 2018). The emergence of radio astronomy in the middle of the last century enabled the detection of many new molecules, and, thanks to the increased sensitivity and angular resolution, this trend has continued at a nearly linear rate ever since. The identification of most of these molecules has been done through an interplay between rotational spectroscopy and radio astronomy by the direct comparison of the emission frequencies from the ISM with laboratory spectroscopic measurements of known species. Since astronomical searches for new molecules are carried out

* Full Tables 2 and 4 and Table A.1 are only available at the CDS via anonymous ftp to cdsarc.u-strasbg. fr (130.79.128.5) or via http://cdsarc.u-strasbg.fr/viz-bin/cat/J/A+A/636/A33

1 CDMS 2020, The Cologne Database for Molecular Spectroscopy: Molecules in the Interstellar Medium or Circumstellar Shells (as of 02/2020), https://cdms.astro.uni-koeln.de/classic/ molecules for even more complicated molecules that show very weak emission spectral signals, it is of utmost importance to provide very precise laboratory data of molecules as likely candidates based on astronomically modelled reactions.

About $15 \%$ of the chemical species detected in the ISM contain a nitrile group. For example, simple molecular species like the $\mathrm{CN}^{-}$anion (Agúndez et al. 2010), alkyl nitriles like $\mathrm{CH}_{3} \mathrm{CN}$ (Solomon et al. 1971) or metal-bearing species like AINC (Ziurys et al. 2002). Surprisingly, the number of dinitriles detected in the ISM is very low compared to the number of nitriles, and, to date, only two - the protonated cyanogen $\left(\mathrm{NCCNH}^{+} ;\right.$Agúndez et al. 2015) and isocyanogen (CNCN; Agúndez et al. 2018) - have been detected in the ISM. This can be easily explained by the fact that the most stable dinitriles, dicyanopolyynes $\mathrm{N} \equiv \mathrm{C}-(\mathrm{C} \equiv \mathrm{C})_{n}-\mathrm{C} \equiv \mathrm{N}$, have no permanent electric dipole moment and therefore cannot be detected through their rotational spectra. In contrast, dinitriles with a saturated hydrocarbon skeleton ended by a $-\mathrm{C} \equiv \mathrm{N}$ group at each edge, that is to say, $\mathrm{N} \equiv \mathrm{C}-\left(\mathrm{CH}_{2}\right)_{n}-\mathrm{C} \equiv \mathrm{N}$, can be considered as good candidates to be observed in the ISM since they present large electric dipole moments. These dinitriles can also be important molecules in the chemistry of planetary bodies, particulary in Titan. Titan's atmosphere is composed of nitrogen (95-98\%), methane $(1.8-5.0 \%)$, hydrogen $(0.1-0.2 \%)$, and carbon monoxide $(0.005 \%)$, with trace amounts of ethane, acetylene, propane, 
ethylene, hydrogen cyanide, cyanoacetylene, carbon dioxide, and water vapor. The photochemistry of these species, initiated by absorption of solar radiation in the vacuum ultraviolet (VUV) region, produces a rich variety of large organic molecules including hydrocarbons, nitriles, or tholins (Sagan \& Khare 1979; Cable et al. 2012).

In order to provide precise data that allow the astronomical detection of dinitriles, laboratory investigations of some of them have been carried out recently. Motiyenko et al. (2019, 2012) observed the rotational spectrum of the simplest molecule of this family of dinitriles, malononitrile $\left(\mathrm{N} \equiv \mathrm{C}-\mathrm{CH}_{2}-\mathrm{C} \equiv \mathrm{N}\right)$ and its isomers isocyanoacetonitrile $\left(\mathrm{N} \equiv \mathrm{C}-\mathrm{CH}_{2}-\mathrm{N} \equiv \mathrm{C}\right)$ and diisocyanomethane $\left(\mathrm{C} \equiv \mathrm{N}-\mathrm{CH}_{2}-\mathrm{N} \equiv \mathrm{C}\right)$ in the frequency range 120 $620 \mathrm{GHz}$. They determined the rotational parameters for these molecules in the ground and in the first vibrational excited states providing accurate frequency predictions to meet astronomical searches for the three molecules. However, their detection in the ISM has not been achieved so far. Succinonitrile, $\mathrm{N} \equiv \mathrm{C}-\mathrm{CH}_{2}-\mathrm{CH}_{2}-\mathrm{C} \equiv \mathrm{N}$, a dinitrile with one more atom carbon in the hydrocarbon skeleton than malononitrile was investigated by Cabezas et al. (2019) in the frequency range 72-116.5 GHz. They identified the ground state and eleven vibrationally excited states and determined their rotational parameters which were employed to unsuccessfully search for succinonitrile in the cold and warm molecular clouds Orion KL, Sgr B2(N), B1-b and TMC-1.

In the present work, we report the rotational investigation of a larger dinitrile, glutaronitrile, with one more atomic carbon in the hydrocarbon skeleton than succinonitrile. To record the rotational spectrum of glutaronitrile, we have employed two different spectroscopic techniques; a high resolution Fourier transform microwave (FTMW) pulsed supersonic jet spectrometer operating in the centimetre wave region and a broadband millimetrewave spectrometer based on radio astronomy receivers with fast Fourier transform backends. The rotational parameters derived from the analysis of the rotational spectrum for the ground state were employed to search for glutaronitrile in the cold and warm molecular clouds Orion KL, Sgr B2(N), B1-b, and TMC-1, using the spectral surveys captured by IRAM $30 \mathrm{~m}$ at $3 \mathrm{~mm}$.

\section{Quantum chemical calculations}

Quantum chemical calculations for the glutaronitrile molecule were carried out at the MP2/cc-pVTZ and CCSD/cc-pVTZ levels of theory using the Molpro 2018.1 quantum chemistry programme package (Werner et al. 2018). Initial searches for minimum energy structures for the glutaronitrile were conducted using the MP2/cc-pVTZ level of theory. The conformational minima found were then optimised at the CCSD/cc-pVTZ level of theory to ascertain their relative stabilities as well as the rotational parameters necessary to predict rotational transition frequencies before the spectral searches. Four conformers were found within an energy window of $4 \mathrm{kcal} \mathrm{mol}^{-1}$. Gaussian 16 programme package (Frisch et al. 2016) was employed to calculate the ${ }^{14} \mathrm{~N}$ electric nuclear quadrupole coupling constants and the centrifugal distortion constants by harmonic and anharmonic frequency calculations at MP2/cc-pVTZ level of theory. The conformers have been labelled according to the dihedral angles of their carbon atom skeleton, in particular the dihedral angles $\angle \mathrm{C}_{1} \mathrm{C}_{2} \mathrm{C}_{3} \mathrm{C}_{4}$ and $\angle \mathrm{C}_{2} \mathrm{C}_{3} \mathrm{C}_{4} \mathrm{C}_{5}$ (see Fig. 1). In this manner, we used the label $g$ or $t$ to denote dihedral angles close to $\pm 60^{\circ}$ or $\pm 180^{\circ}$, respectively, and the conformers were labelled as $g g$, $g t, t t$, and $g g^{\prime}$. The rotational constants, electric dipole moment components and the relative energies for these four species are summarised in Table 1.

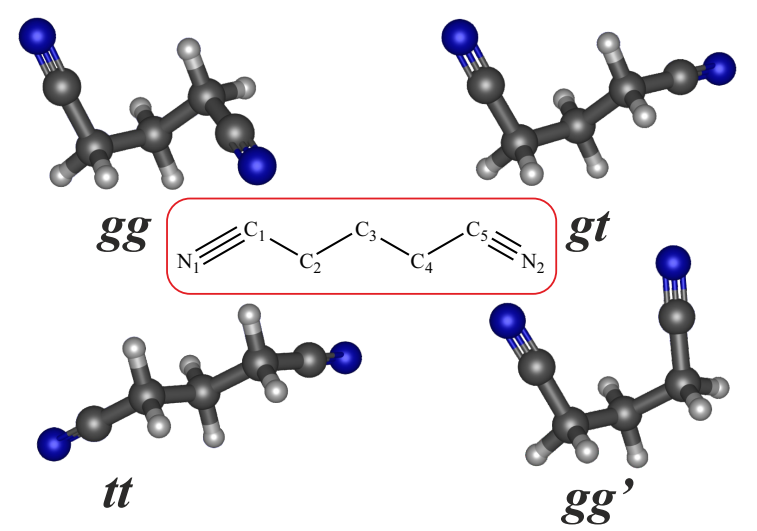

Fig. 1. Molecular structures for the four possible conformers of glutaronitrile obtained from ab initio calculations at the CCSD/cc-pVTZ level of theory.

Table 1. Theoretical spectroscopic constants, electric dipole moment components, and relative energies for the four conformers of glutaronitrile calculated at the CCSD/cc-pVTZ level of theory.

\begin{tabular}{lcccc}
\hline \hline Constants/units & $g g$ & $g t$ & $t t$ & $g g^{\prime}$ \\
\hline$A / \mathrm{MHz}$ & 4767.1 & 7417.1 & 11932.6 & 3461.8 \\
$B / \mathrm{MHz}$ & 1571.6 & 1198.5 & 961.3 & 2282.7 \\
$C / \mathrm{MHz}$ & 1439.5 & 1084.0 & 904.5 & 1454.7 \\
$\left|\mu_{a}\right| / \mathrm{D}^{(a)}$ & 0.0 & 1.6 & 0.0 & 0.0 \\
$\left|\mu_{b}\right| / \mathrm{D}$ & 4.4 & 3.5 & 3.9 & 7.1 \\
$\left|\mu_{c}\right| / \mathrm{D}$ & 0.0 & 1.3 & 0.0 & 0.7 \\
$\Delta \mathrm{E} / \mathrm{cm}^{-1}$ & 0.0 & 247.7 & 587.3 & 1363.4 \\
\hline
\end{tabular}

Notes. ${ }^{(a)} 1 \mathrm{D} \approx 3.3356 \times 10^{-30} \mathrm{C} \cdot \mathrm{m}$.

\section{Experimental}

Firstly, the rotational spectrum of glutaronitrile was investigated using a Balle-Flygare (Balle \& Flygare 1981) narrow-band-type FTMW spectrometer (Cabezas et al. 2016) operating in the frequency region of 4-40 GHz. A commercial sample of glutaronitrile (Sigma-Aldrich, $99 \%$, b.p. $282^{\circ} \mathrm{C}$ ) was used without any further purification. A small amount of glutaronitrile sample was placed in a liquid reservoir which was heated at around $75^{\circ} \mathrm{C}$ to obtain sufficient vapor pressure. A flow of Ar at a pressure of 0.8 bar was passed through the reservoir, placed immediately before the pulsed valve. In this way, the highly diluted mixture of glutaronitrile in Ar was supersonically expanded between the two mirrors of the Fabry-Pérot resonator cavity through a pulsed valve, which is arranged parallel to the resonator in order to resolve small hyperfine splittings. The glutaronitrile molecules are then rapidly cooled to a rotational temperature of $\sim 2 \mathrm{~K}$ to be probed by FTMW spectroscopy. The estimated accuracy of the frequency measurements is better than $3 \mathrm{kHz}$, and the resolution better than $5 \mathrm{kHz}$.

In the second part of the experiment, the emission rotational spectrum of glutaronitrile was recorded from 72 to $116.5 \mathrm{GHz}$ at room temperature using the GACELA (GAS CEll for Laboratory Astrophysics) spectrometer constructed at the Yebes Observatory (Spanish National Geographic Institute (IGN)). The detailed description can be found in Cernicharo et al. (2019). The broadband high resolution rotational spectrometer is equipped with radio receivers similar to those used in radio astronomy to search for molecular emission in space. The receivers are equipped with $16 \times 2.5 \mathrm{GHz}$ fast Fourier transform spectrometers (FFTs) with a spectral resolution of $38.14 \mathrm{kHz}$ allowing the observation of the rotational transitions in the $W$ band $(72-116.5 \mathrm{GHz})$. The spectrometer's cell consists of a stainless steel cylinder of 
$890 \mathrm{~mm}$ length and $490 \mathrm{~mm}$ diameter positioned with the long axis horizontally. The glutaronitrile sample was placed into a Pyrex $^{\mathrm{TM}}$ vacuum Schlenk, which was heated to $80^{\circ} \mathrm{C}$ using a dry heating tape and a PID (proportional integral derivative) temperature controller. In order to avoid sample condensation during the experiment, the temperature of the injection line was kept at $80^{\circ} \mathrm{C}$. Prior to the sample introduction, the pressure inside the vacuum chamber was $2.0 \times 10^{-4}$ mbar and during the experiment, carried out in continuous flow mode, the pressure was kept at $7.0 \times 10^{-3}$ mbar, because higher pressures produce undesirable line broadenings. With the selected working pressure, the rotational lines of glutaronitrile have a HWHM of $0.3-0.45 \mathrm{MHz}$, which is well adapted to frequency measurements with high accuracy. In all these experiments, frequency switching with a throw of $25 \mathrm{MHz}$ was selected as the observing procedure.

\section{Rotational spectrum and analysis}

Glutaronitrile can adopt four conformers in the gas phase, being the $g g$ species, the global minimum, and thus the most probable candidate to be detected through its rotational spectrum. We used the theoretical rotational constants from Table 1 to predict the frequency transitions for this conformer in the 6 to $20 \mathrm{GHz}$ interval. This conformer is a near prolate molecule that has a $C_{2}$ symmetry axis, which coincides with its molecular $b$ axis, and thus it has only one dipole moment component. We found $b$-type $R$-branch rotational transitions with $K_{a}=0,1$ very close to the predictions. They were assigned to improve the transition frequency predictions and search for new ones. In this manner, we extended our measurements to $b$-type $R$-branch transitions with $K_{a}=2$ and $b$-type $Q$-branch transitions with $K_{a}=1,2$ and 3. Each rotational transition is split into several hyperfine components because of the nuclear quadrupole coupling effects produced by the presence of two equivalent nitrogen atoms, as can be seen in Fig. 2 for the $3_{3,1}-3_{2,2}$ rotational transition. The interaction at the nuclei of these quadrupole moments with the electric field gradient created by the rest of the molecular charges causes the coupling of the nuclear spin moments to the overall rotational momentum (Gordy \& Cook 1984). In total we observed 228 hyperfine components corresponding to 24 pure rotational transitions (see Table 2). Rotational, centrifugal, and nuclear quadrupole coupling constants were determined fitting the transition frequencies, using the SPFIT programme (Pickett 1991), to a Watson's A-reduced ${ }^{1}$ A' Hamiltonian for asymmetric top molecules, with the following form (Watson 1977): $H=H_{\mathrm{R}}+H_{\mathrm{Q}}$, where $H_{\mathrm{R}}$ contains rotational and centrifugal distortion parameters and $H_{\mathrm{Q}}$ the quadrupole coupling interactions. The energy levels involved in each transition are labelled with the quantum numbers $J, K_{a}, K_{c}, I$, and $F$, where $I=I_{N_{1}}+I_{N_{2}}$ and $F=J+I$, where $I=0,1$, and 2 . The analysis rendered the experimental constants listed in Table 3 together with theoretical data. Comparison of the experimentally determined rotational constants with those predicted ab initio leads to the unambiguous identification of conformer $g g$. The calculated constants of Table 3 show deviations from experimental values lower than 0.7 $\%$, indicating that the calculated ab initio geometry is fairly accurate. In spite of the fact that larger discrepancies can be found between the experimental and theoretical centrifugal distortion constants, the accordance can be considered satisfactory since the estimation procedure of these parameters is not trivial. Finally, it can be seen that the nuclear quadrupole coupling constants agree very well with those predicted theoretically.

Once the $g g$ conformer was assigned, we looked for the rotational transitions of higher energy conformers, in particular $g t$

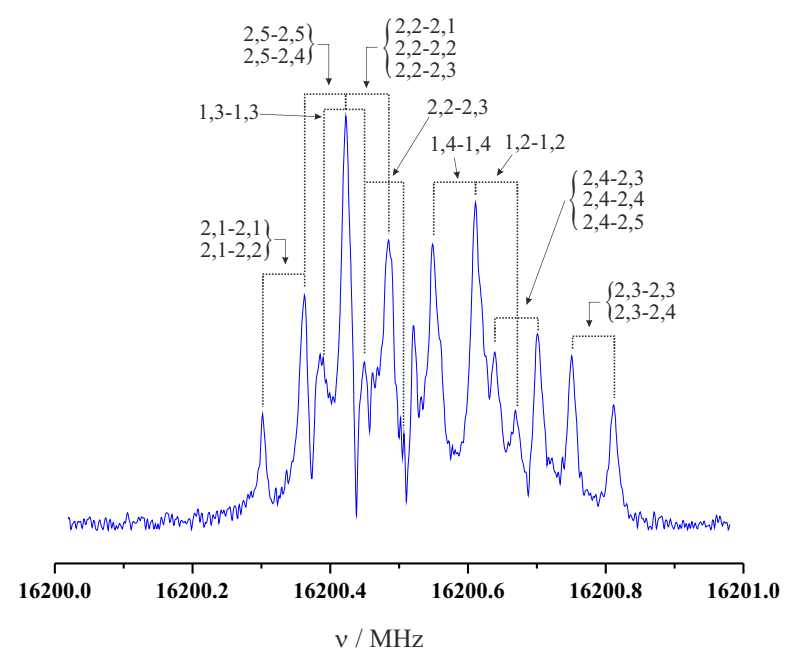

Fig. 2. $3_{3,1}-3_{2,2}$ rotational transition of $g g$ conformer of glutaronitrile showing the multiple hyperfine components completely resolved by FTMW spectrometer. The coaxial arrangement of the adiabatic expansion and the resonator axis produces an instrumental Doppler doubling, which is marked with the dashed lines. The resonances frequencies are calculated as the average of the two Doppler components. Each hyperfine component is labelled with the corresponding values of quantum numbers $I^{\prime}, F^{\prime}-I^{\prime \prime}, F^{\prime \prime}$.

species. According to its relative energy, its estimated population (at the cell's temperature, $300 \mathrm{~K}$ ) is $\sim 1 / 3$ of that of $g g$ conformer but taking into account the values of dipole moment components predicted by ab initio calculations, the expected intensity of its rotational transitions is $\sim 1 / 5$ of those observed for $g g$ conformer. We scanned the predicted frequency regions, but no lines attributable to $g t$ conformer were found. Given the good signalto-noise ratio for the rotational transitions of the $g g$ conformer observed by FTMW spectroscopy, it seems that $g t$ should be also detected. The absence of the $g t$ conformer in the supersonic jet can be associated with a collisional relaxation of this conformer to the $g g$ conformer through a low-energy interconversion barrier. This process takes place in the early stages of the supersonic expansion by collisions between the different conformers and the buffer gas molecules (Ruoff et al. 1990). It has been shown that collisional removal of a higher energy conformer is possible when the isomerisation barrier is around 500-600 $\mathrm{cm}^{-1}$ and Ar is used as carrier gas (Cabezas et al. 2018). To gain some insight, we calculated the potential energy profile between the $g t$ and $g g$ conformers by scanning the dihedral angle $\angle \mathrm{C}_{1} \mathrm{C}_{2} \mathrm{C}_{3} \mathrm{C}_{4}$. An energy barrier of $698 \mathrm{~cm}^{-1}$ was found, at the CCSD/cc-pVTZ level of theory, suggesting a collisional relaxation, at least partial, from $g t$ to $g g$ conformer. This fact makes the $g t$ conformer population smaller than the expected one, preventing the observation of its rotational transitions.

Using the rotational and centrifugal distortion constants determined by FTMW measurements, we predicted the transition frequencies for the $g g$ conformer of glutaronitrile in the $\mathrm{mmW}$ frequency region. An initial assignment was obtained simply by locating the $b$-type $R$-branch transitions with $K_{a}=0,1$ as those depicted in Fig. 3. Measurements were extended to $b$-type $R$-branch rotational transitions up to $K_{a}=3$ and $Q$-branch transitions with $K_{a}=17,18$. A total of 89 rotational transitions, see Table 4, were observed in the $\mathrm{mmW}$ range and fitted (Pickett 1991) to a Watson's A-reduced ${ }^{1} \mathrm{~A}^{\prime}$ Hamiltonian. The analysis rendered the experimental data listed in Table 3. In addition to the rotational and quartic centrifugal distortion constants previously determined by FTMW measurements, the sextic centrifugal 
Table 2. Rotational transitions for glutaronitrile observed by FTMW spectroscopy.

\begin{tabular}{lcccccccccccc}
\hline \hline$J^{\prime(a)}$ & $K_{a}^{\prime}$ & $K_{c}^{\prime}$ & $I^{\prime}$ & $F^{\prime}$ & $J^{\prime \prime}$ & $K_{a}^{\prime \prime}$ & $K_{c}^{\prime \prime}$ & $I^{\prime \prime}$ & $F^{\prime \prime}$ & $\begin{array}{c}\text { Freq. meas. } \\
(\mathrm{MHz})\end{array}$ & $\begin{array}{c}\text { Uncert. } \\
(\mathrm{MHz})\end{array}$ & $\begin{array}{c}\text { Obs.calc. }{ }^{(d)} \\
(\mathrm{MHz})\end{array}$ \\
\hline 2 & 2 & 0 & 2 & 4 & 2 & 1 & 1 & 2 & 4 & 9523.644 & 0.005 & 0.000 \\
2 & 2 & 0 & 1 & 3 & 2 & 1 & 1 & 1 & 3 & 9523.754 & 0.005 & -0.000 \\
2 & 2 & 0 & 1 & 1 & 2 & 1 & 1 & 1 & 1 & 9523.848 & 0.005 & 0.001 \\
2 & 2 & 0 & 2 & 3 & 2 & 1 & 1 & 2 & 3 & 9523.866 & 0.005 & 0.001 \\
2 & 2 & 0 & 2 & 2 & 2 & 1 & 1 & 2 & 2 & 9523.943 & 0.005 & 0.001 \\
2 & 2 & 1 & 0 & 2 & 2 & 1 & 2 & 0 & 2 & 9919.754 & 0.005 & -0.000 \\
2 & 2 & 1 & 1 & 2 & 2 & 1 & 2 & 1 & 2 & 9919.807 & 0.005 & -0.003 \\
2 & 2 & 1 & 2 & 4 & 2 & 1 & 2 & 2 & 4 & 9919.886 & 0.005 & -0.003 \\
2 & 2 & 1 & 1 & 3 & 2 & 1 & 2 & 1 & 3 & 9920.044 & 0.005 & -0.000 \\
2 & 2 & 1 & 1 & 1 & 2 & 1 & 2 & 1 & 1 & 9920.178 & 0.005 & 0.004 \\
2 & 2 & 1 & 2 & 3 & 2 & 1 & 2 & 2 & 3 & 9920.199 & 0.005 & -0.001 \\
2 & 2 & 1 & 2 & 2 & 2 & 1 & 2 & 2 & 2 & 9920.309 & 0.005 & 0.001 \\
2 & 1 & 2 & 2 & 1 & 1 & 0 & 1 & 2 & 1 & 9103.868 & 0.005 & 0.002 \\
2 & 1 & 2 & 1 & 1 & 1 & 0 & 1 & 1 & 1 & 9103.879 & 0.005 & 0.002 \\
2 & 1 & 2 & 0 & 2 & 1 & 0 & 1 & 2 & 1 & 9103.891 & 0.005 & 0.001 \\
2 & 1 & 2 & 2 & 3 & 1 & 0 & 1 & 2 & 3 & 9103.926 & 0.005 & -0.001 \\
2 & 1 & 2 & 1 & 3 & 1 & 0 & 1 & 1 & 2 & 9104.052 & 0.005 & -0.003 \\
\hline
\end{tabular}

Notes. ${ }^{(a)} J^{\prime}, K_{a}^{\prime}, K_{c}^{\prime}, I^{\prime}$, and $F^{\prime}$ represent the quantum numbers associated with the upper rotational level. $J^{\prime \prime}, K_{a}^{\prime \prime}, K_{c}^{\prime \prime}, I^{\prime \prime}$, and $F^{\prime \prime}$ represent the quantum numbers associated with the lower rotational level. ${ }^{(b)}$ Observed experimental frequency. ${ }^{(c)}$ Uncertainty in the observed frequency. ${ }^{(d)}$ Difference between the observed frequency and that derived from the fit. The full table is available at the CDS.

Table 3. Molecular constants for glutaronitrile.

\begin{tabular}{lcccc}
\hline \hline Constants/units & $F T M W$ fit & Global fit & mmW fit & Theory ${ }^{(a)}$ \\
\hline$A / \mathrm{MHz}$ & $4756.13738(24)$ & $4756.1397(30)$ & $4755.60(11)$ & 4767.1 \\
$B / \mathrm{MHz}$ & $1582.79295(64)$ & $1582.7606(25)$ & $1582.7530(84)$ & 1571.6 \\
$C / \mathrm{MHz}$ & $1449.29522(55)$ & $1449.27921(65)$ & $1449.28150(85)$ & 1439.5 \\
$\Delta_{J} / \mathrm{kHz}$ & $2.4036(64)$ & $2.3447(16)$ & $2.3555(52)$ & 2.2839 \\
$\Delta_{K} / \mathrm{kHz}$ & $56.221(24)$ & $59.787(41)$ & $57.89(39)$ & 35.8181 \\
$\Delta_{J K} / \mathrm{kHz}$ & $-19.905(21)$ & $-20.4772(93)$ & $-20.486(11)$ & -21.7187 \\
$\delta_{J} / \mathrm{kHz}$ & $0.5920(32)$ & $0.54303(77)$ & $0.5477(25)$ & 0.3213 \\
$\delta_{K} / \mathrm{kHz}$ & $4.54(26)$ & $4.282(45)$ & $4.78(14)$ & 5.3779 \\
$\Phi_{K} / \mathrm{Hz}$ & - & $6.680(87)$ & $4.40(47)$ & 1.3457 \\
$\Phi_{K J} / \mathrm{Hz}$ & - & $-1.834(17)$ & $-1.804(21)$ & -1.2627 \\
$\phi_{J K} / \mathrm{Hz}$ & - & $-0.112(17)$ & $-0.110(30)$ & -0.5042 \\
$\chi_{a a} / \mathrm{MHz}$ & $-0.4123(10)$ & - & - & -0.4126 \\
$\chi_{b b} / \mathrm{MHz}$ & $0.0989(15)$ & - & - & 0.1045 \\
$\sigma_{\text {rms }} / \mathrm{kHz}$ & 3.44 & 38.10 & 42.33 & - \\
$\sigma_{\text {wrms }}$ & $0.60^{(b)}$ & $0.76^{(c)}$ & $0.84{ }^{(c)}$ & - \\
$N_{\text {lines }}$ & $228^{(d)}$ & 111 & 89 & - \\
$J_{\min } / J_{\max }$ & $0 / 5$ & $0 / 54$ & $24 / 54$ & - \\
$K_{a, \min } / K_{a, \max }$ & $0 / 3$ & $0 / 18$ & $0 / 18$ & - \\
\hline
\end{tabular}

Notes. Numbers in parentheses represent the derived uncertainty $(1 \sigma)$ of the parameter in units of the last digit. ${ }^{(a)}$ Rotational and nuclear quadrupole coupling constants derived from the optimisation calculations at CCSD/cc-pVTZ level of theory. The distortion constants were obtained from anharmonic frequency MP2/cc-pVTZ calculations. ${ }^{(b)}$ The uncertainty associated with the lines is $5 \mathrm{kHz} .{ }^{(c)}$ The uncertainty associated with the lines is $50 \mathrm{kHz} .{ }^{(d)}$ Number of hyperfine components included in the fit.

distortion constants $\Phi_{K}, \Phi_{K J}$, and $\phi_{J K}$ were determined. The inclusion of these parameters in the fit slightly affects the value of the rest of the floated parameters, as can be seen if data for the FTMW and mmw fits are compared (Table 3).

Collisional relaxation between conformers is not possible in the GACELA experiments since they are not performed in a supersonic expansion environment. Hence, the rotational transitions for $g t$ conformer should be observed in our $\mathrm{mmW}$ spectrum with intensity five times smaller than those for the $g g$ conformer. However, the signal-to-noise ratio of the $g g$ conformer is much smaller in the $\mathrm{mmW}$ region than that found in the FTMW experiments, and then, the rotational lines for $g t$ conformer could not be distinguished from the noise level.
As mentioned before, glutaronitrile has two equivalent ${ }^{14} \mathrm{~N}$ nuclei with a spin of $I=1$, so that nuclear quadrupole coupling hyperfine splittings were observed using the FTMW spectrometer. However, in the $\mathrm{mmW}$ range, all the measured lines were observed as single lines, and no hyperfine splittings could be noticed (Fig. 3) because they are smaller than the experimental broadening of the lines. The rotational spectrum in the $\mathrm{mmW}$ region, yet, shows the influence of nuclear spin statistics on the transition intensities. This is due to the geometry of the molecule, which allows an interchange of identical particles by the rotation around one of the principal axes. The $C_{2 b}$ operation of the $C_{2}$ symmetry point group, which corresponds to the rotation about the $b$ axis by $\pi$ radians, simultaneously exchanges the 
Table 4. Rotational transitions for glutaronitrile observed in the $\mathrm{mmW}$ region.

\begin{tabular}{lcccccccc}
\hline \hline$J^{\prime(a)}$ & $K_{a}^{\prime}$ & $K_{c}^{\prime}$ & $J^{\prime \prime}$ & $K_{a}^{\prime \prime}$ & $K_{c}^{\prime \prime}$ & $\begin{array}{c}\text { Freq. meas. }^{(b)} \\
(\mathrm{MHz})\end{array}$ & $\begin{array}{c}\text { Uncert. }^{(c)} \\
(\mathrm{MHz})\end{array}$ & $\begin{array}{c}\text { Obs.-calc. }{ }^{(d)} \\
(\mathrm{MHz})\end{array}$ \\
\hline 40 & 1 & 40 & 39 & 0 & 39 & 116272.079 & 0.050 & -0.010 \\
40 & 0 & 40 & 39 & 1 & 39 & 116271.949 & 0.050 & 0.016 \\
39 & 2 & 38 & 38 & 1 & 37 & 114711.079 & 0.050 & -0.038 \\
39 & 1 & 38 & 38 & 2 & 37 & 114700.167 & 0.050 & -0.033 \\
39 & 1 & 39 & 38 & 0 & 38 & 113397.853 & 0.050 & 0.018 \\
39 & 0 & 39 & 38 & 1 & 38 & 113397.669 & 0.050 & 0.041 \\
38 & 2 & 37 & 37 & 1 & 36 & 111839.559 & 0.050 & -0.023 \\
38 & 1 & 37 & 37 & 2 & 36 & 111824.587 & 0.050 & -0.021 \\
38 & 1 & 38 & 37 & 0 & 37 & 110522.432 & 0.050 & 0.023 \\
38 & 0 & 38 & 37 & 1 & 37 & 110522.139 & 0.050 & 0.023 \\
36 & 1 & 36 & 35 & 0 & 35 & 104768.151 & 0.050 & -0.009 \\
36 & 0 & 36 & 35 & 1 & 35 & 104767.553 & 0.050 & -0.020 \\
\hline
\end{tabular}

Notes. ${ }^{(a)} J^{\prime}, K_{a}^{\prime}$, and $K_{c}^{\prime}$ represent the quantum numbers associated with the upper rotational level. $J^{\prime \prime}, K_{a}^{\prime \prime}$, and $K_{c}^{\prime \prime}$ represent the quantum numbers associated with the lower rotational level. ${ }^{(b)}$ Observed experimental frequency. ${ }^{(c)}$ Uncertainty in the observed frequency. ${ }^{(d)}$ Difference between the observed frequency and that derived from the fit. The full table is available at the CDS.

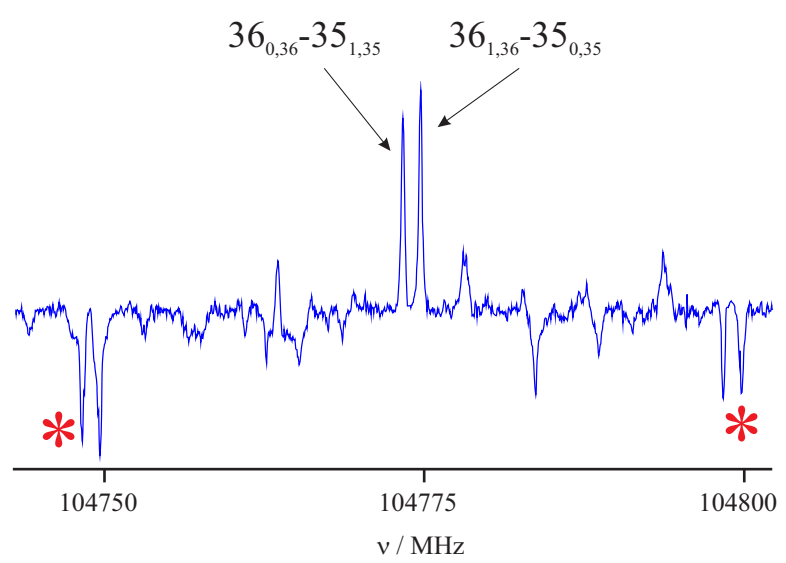

Fig. 3. Section of broadband rotational spectrum of glutaronitrile showing the $R$-branch rotational transitions $36_{0,36}-35_{1,35}$ and $36_{1,36}-35_{0,35}$ of $g g$ conformer of glutaronitrile. The negative features marked with an asterisk are produced by the folding of the frequency switched data and appear at $\pm 25 \mathrm{MHz}$ of each rotational transition.

positions of three non-equivalent pairs of hydrogen (fermions with $I=1 / 2$ ) and two nitrogen atoms (bosons with $I=1$ ) in the molecule. Fermi-Dirac statistics must be considered due to the presence of three pairs of fermions and the bosons. Hence, the total wavefunction, expressed as $\psi_{\text {tot }}=\psi_{\text {ele }} \psi_{\text {vib }} \psi_{\text {rot }} \psi_{\mathrm{ns}}$, must be antisymmetric with respect to the $C_{2 b}$ operation. The corresponding wavefunctions $\psi_{\text {ele }}$ and $\psi_{\text {vib }}$ for the ground electronic and vibrational states are symmetric. The parity of the rotational wavefunction $\psi_{\text {rot }}$ depends on the $K_{a}$ and $K_{c}$ values, and it is symmetric for the levels with $K_{a}+K_{c}=$ even, while for the levels with $K_{a}+K_{c}=$ odd it is antisymmetric. Hence, to satisfy Fermi-Dirac statistics, symmetric and antisymmetric nuclear spin functions must be combined with antisymmetric and symmetric rotational wavefunctions, respectively. As shown in Bunker \& Jensen (1998), the total nuclear statistics weights are $\left(2 I_{H}+1\right)^{2}\left(2 I_{H}+1\right)^{2}\left(2 I_{H}+1\right)^{2}\left(2 I_{N}+1\right)^{2}=576$, and the nuclear statistical weight for the rotational levels with $K_{a}+K_{c}=$ even and odd is 23 and 25 , respectively. Figure 3 shows the $36_{0,36}-35_{1,35}$ and $36_{1,36}-35_{0,35}$ rotational transitions and their relative intensity ratio of 23-to-25.

A combined fit using all the transition frequencies from both FTMW and mmW measurements was done. The spectroscopic
Table 5. Rotational partition functions for glutaronitrile at different temperatures.

\begin{tabular}{lc}
\hline \hline Temperature/K & $Q_{r}$ \\
\hline 300.000 & 223332.4 \\
225.000 & 158807.8 \\
150.000 & 92210.4 \\
75.000 & 33259.6 \\
37.500 & 11747.9 \\
18.750 & 4152.4 \\
9.375 & 1469.2 \\
\hline
\end{tabular}

constants derived for the $g g$ conformer are shown in Table 3 together with the other fits and the theoretical calculations. In order to search for glutaronitrile in the space, we implemented the constants derived from the combined fit into the MADEX code (Cernicharo 2012) and simulated the spectra of glutaronitrile at higher frequencies. The frequency predictions for glutaronitrile up to $300 \mathrm{GHz}$ calculated at $T=300 \mathrm{~K}$ are given in Table A.3 available at CDS. To estimate the column density of glutaronitrile, we used the partition function values shown in Table 5, which were calculated including rotational levels at maximum value of $J=90$. The results of the astronomical search are shown in the next section.

\section{Search for glutaronitrile in space}

Using the rotational parameters for the observed $g g$ conformer of glutaronitrile and the MADEX code (Cernicharo 2012), we searched for it in space. We searched for glutaronitrile in the two high-mass star-forming regions, Orion KL and Sagittarius (Sgr) B2 using the IRAM $30 \mathrm{~m}$ data at $3 \mathrm{~mm}$ provided by Tercero et al. (2010) and Belloche et al. (2013), respectively. In addition, we extended our search to the starless core in the Taurus Molecular Cloud (TMC-1) and the cold dark cloud Barnard 1 (B1-b) using $3 \mathrm{~mm}$ data of the IRAM $30 \mathrm{~m}$ telescope (Marcelino et al. 2007; Cernicharo 2012). We focused on data at frequencies below $200 \mathrm{GHz}$ to assess a potential detection and prevent non-reliable frequency predictions.

Firstly, we searched for glutaronitrile using the IRAM $30 \mathrm{~m}$ data at $3 \mathrm{~mm}$ of TMC-1 and B1-b sources. For these cold cores, we focused the search on unresolved doublets with energies of the upper level of about $20 \mathrm{~K}$ such as $10_{9,2}-9_{8,1}$ and $10_{9,1}-9_{8,2}$ 
Table 6. Physical parameters of the considered cloud cores in the astronomical search of glutaronitrile.

\begin{tabular}{|c|c|c|c|c|c|c|c|c|}
\hline Source & $\begin{array}{c}\text { Coordinates } \\
\text { J2000.0 }\end{array}$ & $\begin{array}{l}\operatorname{HPBW}^{(a)} \\
\left(^{\prime \prime}\right)\end{array}$ & $\begin{array}{c}\text { Frequencies }{ }^{(b)} \\
(\mathrm{GHz})\end{array}$ & $\begin{array}{c}v_{\mathrm{LSR}}{ }^{(c)} \\
\left(\mathrm{km} \mathrm{s}^{-1}\right)\end{array}$ & $\begin{array}{c}\Delta v_{F W H M}{ }^{(d)} \\
\left(\mathrm{km} \mathrm{s}^{-1}\right)\end{array}$ & $\begin{array}{c}d_{\text {sou }}(e) \\
\left({ }^{\prime \prime}\right)\end{array}$ & $\begin{array}{c}T_{\text {rot }}(f) \\
(\mathrm{K})\end{array}$ & $\begin{array}{c}N^{(\mathrm{g})} \\
\times 10^{15}\left(\mathrm{~cm}^{-2}\right)\end{array}$ \\
\hline Orion KL & $\begin{array}{c}\alpha=5^{\mathrm{h}} 35^{\mathrm{m}} 14 \mathrm{~s} .5 \\
\delta=-05^{\circ} 22^{\prime} 30^{\prime \prime} .0\end{array}$ & $30-21$ & $80-115.5$ & $\begin{array}{l}5.0 \\
5.0 \\
3.0 \\
3.0\end{array}$ & $\begin{array}{l}6.0 \\
6.0 \\
20 \\
20\end{array}$ & $\begin{array}{l}10 \\
15 \\
10 \\
15\end{array}$ & $\begin{array}{c}320 \\
100 \\
200 \\
90\end{array}$ & $\begin{aligned} & \leq(1.0 \pm 0.5) \\
\leq & (0.50 \pm 0.25) \\
\leq & (0.20 \pm 0.10) \\
\leq & (0.20 \pm 0.10)\end{aligned}$ \\
\hline Sgr B2(N) & $\begin{array}{c}\alpha=17^{\mathrm{h}} 47^{\mathrm{m}} 20.0 \\
\delta=-28^{\circ} 22^{\prime} 19^{\prime \prime} 0\end{array}$ & $30-21$ & $80-115.5$ & $\begin{array}{l}63 \\
73 \\
53 \\
\end{array}$ & $\begin{array}{c}6.5 \\
6.5 \\
8 \\
\end{array}$ & $\begin{array}{l}2.7 \\
2.7 \\
2.7 \\
\end{array}$ & $\begin{array}{l}200 \\
200 \\
200 \\
\end{array}$ & $\begin{array}{l}\leq(100 \pm 50) \\
\leq(50 \pm 25) \\
\leq(50 \pm 25)\end{array}$ \\
\hline B1-b & $\begin{array}{l}\alpha=03^{\mathrm{h}} 33^{\mathrm{m}} 20^{\mathrm{s}} 0 \\
\delta=31^{\circ} 07^{\prime} 34^{\prime \prime} .0\end{array}$ & $30-21$ & $82.5-115.5$ & 6.7 & 0.7 & 60 & 12 & $\leq(0.002 \pm 0.001)$ \\
\hline TMC-1 & $\begin{array}{l}\alpha=04^{\mathrm{h}} 41^{\mathrm{m}} 41^{\mathrm{s}} 9 \\
\delta=25^{\circ} 41^{\prime} 27^{\prime \prime} \cdot 0\end{array}$ & $30-21$ & $82.5-115.5$ & 6.0 & 0.7 & 60 & 10 & $\leq(0.002 \pm 0.001)$ \\
\hline
\end{tabular}

Notes. ${ }^{(a)}$ Half power beam width. ${ }^{(b)}$ Range of frequencies considered in the analysis. ${ }^{(c)}$ Radial velocity with respect the Local Standard of Rest. ${ }^{(d)}$ Full width at half maximum. ${ }^{(e)}$ Source diameter assuming a uniform disc-type model. ${ }^{(f)}$ Rotational temperature. ${ }^{(g)}$ Column density for glutaronitrile.

at $85307 \mathrm{MHz}$ and $11_{9,3}-10_{8,2}$ and $11_{9,2}-10_{8,3}$ at $88347 \mathrm{MHz}$ We did not detect glutaronitrile above the $3 \sigma$ detection limit of our data. The derived upper limits for glutaronitrile column densities, considering local thermodynamic equilibrium (LTE), and the assumed physical parameters are summarised in Table 6. For the high-mass star-forming regions, we built a synthetic spectrum of glutaronitrile adopting the physical parameters derived for $\mathrm{CH}_{2} \mathrm{CHCN}$ by López et al. (2014) in Orion KL, and those derived for $\mathrm{CH}_{3} \mathrm{CN}$ by Belloche et al. (2013) in Sgr B2.

These results are not totally unexpected, since in a previous work (Cabezas et al. 2019) we were not able to detect the simpler succinonitrile. However, the negative result for the detection of succinonitrile may be related to the fact that the search was limited to the gauche conformer which is $321 \mathrm{~cm}^{-1}$ above the most stable conformer, the trans one, which is a non-polar species. Therefore, the search for a more complex species like glutaronitrile is required in order to constrain the production of complex nitriles in space. A general discussion related to the source selection and the production of dinitriles in space can be found in our previous work on succinonitrile (Cabezas et al. 2019). Here, we only provide upper limits for the glutaronitrile column density in these sources. Like in the case of succinonitrile, the derived upper limits are only a factor $\leq 10$ lower than the column density derived for $\mathrm{CH}_{3} \mathrm{CN}$ in Orion KL (López et al. 2014) and Sgr B2 (Belloche et al. 2013). This suggests that the high level of line confusion in the considered surveys produces very high upper limits for the column densities of the non-detected species. We also note that the derived upper limits are only a factor of 1.5 and 5 lower than those obtained for the total column densities of succinonitrile (including the lowest energy conformer) provided by Cabezas et al. (2019) for Orion KL and Sgr B2, respectively.

\section{Conclusions}

The present work reports the investigation of the rotational spectra of a new dinitrile, glutaronitrile. The microwave and millimetre-wave spectra were measured using two different spectroscopic techniques: a FTMW spectrometer at low frequencies, and a broadband millimetre-wave spectrometer in the frequency range $72-116.5 \mathrm{GHz}$. From the analysis of the spectra, we obtain accurate rotational parameters for the most stable conformation, named the $g g$ species, which were used to unsuccessfully search for glutaronitrile in the cold and warm molecular clouds Orion KL, Sgr B2(N), B1-b, and TMC-1, using the spectral surveys captured by IRAM $30 \mathrm{~m}$ at $3 \mathrm{~mm}$.
Acknowledgements. We thank the European Research Council for funding support under Synergy Grant ERC-2013-SyG, G.A. 610256 (NANOCOSMOS). CB thanks to Ministerio de Ciencia, Innovación y Universidades for a Juan de la Cierva postdoctoral fellowship (FJCI-2016-27983). YE thanks to the Ministry of Science and Technology of Taiwan for the Grant MOST 104-2113-M-009020. This work was also based on observations carried out with the IRAM $30 \mathrm{~m}$ telescope. IRAM is supported by INSU/CNRS (France), MPG (Germany), and IGN (Spain)

\section{References}

Agúndez, M., Cernicharo, J., Guélin, M., et al. 2010, A\&A, 517, L2 Agúndez, M., Cernicharo, J., de Vicente, P., et al. 2015, A\&A, 579, L10 Agúndez, M., Marcelino, N., \& Cernicharo, J. 2018, ApJ, 861, L22

Belloche, A., Müller, H. S. P., Menten, K. M., Schilke, P., \& Comito, C. 2013, A\&A, 559, A47

Bunker, P. R., \& Jensen, P. 1998, Molecular Symmetry and Spectroscopy, 2nd edn. (Ottawa: NRC Research Press)

Balle, T. J., \& Flygare, W. H. 1981, Rev. Sci. Instrum., 52, 33

Cabezas, C., Guillemin, J.-C., \& Endo, Y. 2016, J. Chem. Phys., 145, 184304 Cabezas, C., Guillemin, J.-C., \& Endo, Y. 2018, J. Chem. Phys., 149, 084309

Cabezas, C., Bermúdez, C., Gallego, J. D., et al. 2019, A\&A, 629, A35

Cable, M. L., Hörst, S. M., \& Hodyss, R. 2012, Chem. Rev., 112, 1882

Cernicharo, J. 2012, in ECLA-2011: Proc. of the European Conference on Laboratory Astrophysics, eds. C. Stehl, C. Joblin, \& L. d'Hendecourt (Cambridge: Cambridge Univ. Press), EAS Pub. Series, 251, https:// nanocosmos. iff. csic.es/?pageid $=1619$

Cernicharo, J., Gallego, J. D., López-Pérez, J. A., et al. 2019, A\&A, 626, A34

Dunham, T. J. 1937, PASP, 49, 26

Frisch, M. J., Trucks, G. W., Schlegel, H. B., et al. 2016, Gaussian 16, revision A.03

Gordy, W., \& Cook, R. 1984, Microwave Molecular Spectra, Techniques of Chemistry (New York: Wiley)

López, A., Tercero, B., Kisiel, Z., et al. 2014, A\&A, 572, A44

McGuire, B. A., Burkhardt, A. M., Kalenskii, S., et al. 2018, Sci, 359, 202

McKellar, A. 1940, PASP, 52, 187

Marcelino, N., Cernicharo, J., Agúndez, M., et al. 2007, ApJ, 665, L127

Motiyenko, R. A., Margulés, L., \& Guillemin, J.-C. 2012, A\&A, 544, A82

Motiyenko, R. A., Armieieva, I. A., Margulés, L., et al. 2019, A\&A, 623, A162

Pickett, H. M. 1991, JMoSp, 148, 371

Ruoff, R. S., Klots, T. D., Emilsson, T., \& Gutowsky, H. S. 1990, J. Chem. Phys., 93, 3142

Sagan, C., \& Khare, B. N. 1979, Nature, 277, 102

Solomon, P. M., Jefferts, K. B., Penzias, A. A., \& Wilson, R. W. 1971, ApJ, 168, L107

Swings, P., \& Rosenfeld, L. 1937, ApJ, 86, 483

Tercero, B., Cernicharo, J., Pardo, J. R., \& Goicoechea, J. R. 2010, A\&A, 517, A96

Watson, J. K. G. 1977, in Vibration Spectra and Structure (Amsterdam: Elsevier), ed. J. Durig, 6, 1

Werner, H.-J., Knowles, P. J., Knizia, G., et al. 2018, MOLPRO, version 2018.1. Ziurys, L. M., Savage, C., Highberger, J. L., et al. 2002, ApJ, 564, L45 